\title{
Estimación y localización de fugas en una red de tuberías de agua potable usando algoritmos genéticos
}

\author{
Estimation and Location of Leaks in a Pipe Water Network \\ Using Genetic Algorithms
}

\author{
Fuentes-Mariles O.A. \\ Instituto de Ingeniería \\ Universidad Nacional Autónoma de México \\ E-mail:ofm@pumas.iingen.unam.mx \\ Palma-Nava A. \\ Instituto de Ingeniería \\ Universidad Nacional Autónoma de México \\ E-mail:apn@pumas.iingen.unam.mx
}

\author{
Rodríguez-Vázquez K. \\ Instituto de Matemáticas Aplicadas \\ Universidad Nacional Autónoma de México \\ E-mail:katya@uxdea4.iimas.unam.mx
}

Información del artículo: recibido: mayo de 2007, aceptado: noviembre de 2010

\section{Resumen}

Las fugas de agua potable en las redes de distribución producen desperdicio de agua, reducen la eficiencia de las redes y generan una pérdida económica a los organismos operadores del sistema de distribución de este tipo de líquido. La detección de fugas en una red de tuberías es complicada, ya que en su mayoría no se encuentran visibles. Para reducirlas es necesario contar con procedimientos e instrumentos especiales para localizarlas y eliminarlas. En este artículo se expone un método para detectar fugas a lo largo de las tuberías. Con él se determina el caudal de las mismas y la posición donde ocurren en las tuberías de una red cerrada. Se considera que la red funciona en flujo permanente a presión y que sólo existe una fuga por cada tubería de la red. El procedimiento propuesto se basa en las mediciones de la presión en la unión de las tuberías, el conocimiento de las características de la red y la estimación de las demandas de caudal. Se emplea la teoría de la computación evolutiva, en particular, un algoritmo genético simple, como mecanismo de búsqueda de la solución óptima. Este proceso es iterativo hasta disminuir el error entre las cargas medidas y las calculadas con este método. Se incluye un ejemplo a partir de las mediciones obtenidas en laboratorio para mostrar la bondad del método y la forma de aplicarlo.

\section{Descriptores}

- fugas

- caudales de fuga

- detección de fugas

- redes de tuberías

- suministro de agua

- distribución de agua potable

- algoritmos genéticos 


\begin{abstract}
Leakage in distribution pipe networks for drinkable water produces waste of water resources, reducing the network efficiency and generating an economic loss to the official organisms that control the network distribution system. Leakages detection in a pipe network is a complicated task due to these leakages are not visible. In order to reduce them, it is necessary to have procedures and special equipment to locate and repair these leakages. In this paper, a method for leakages detection along a pipe network is proposed. The aim is to determine the flow and position where the water is leaking in pipes of a closed network. It is also considered that the network, in this case, works under permanent flow pressure and there is only a leakage presented in each pipe of the network. The method proposed here is based on pressure measured at the union of the pipes, the characteristics of the network and the estimation of flow demands. An Evolutionary Computation algorithm, specifically a Genetic Algorithm, is used as the mechanism for searching an optimal solution. This process is iterative until the error between measured "heads" and those calculated by means of a genetic algorithm is substantially reduced. An example is also presented based on measurements from a laboratory test bed in order to show the robustness of the proposed method and its application.
\end{abstract}

\section{Introducción}

Una fuga es una salida de agua no controlada en cualquiera de los componentes del sistema de distribución de agua potable; con mayor frecuencia ocurren en uniones de tuberías, codos, roturas de conductos y válvulas.

En los sistemas de conducción de agua a presión es común que se presenten fugas de este líquido. Las fugas pueden deberse al agrietamiento transversal, aplastamiento o agrietamiento longitudinal de las tuberías, la corrosión, el mal junteo de los tubos o la falla de las válvulas que pueden incrementar las fugas en una red.

En algunos países, las fugas de los sistemas de abastecimiento de agua llegan a ser del 50\% de la cantidad requerida por los habitantes para satisfacer sus necesidades hídricas; esto implica pérdidas económicas de importancia y un mal aprovechamiento de los recursos naturales. En Malasia el porcentaje de las fugas es del orden del 40\%, en Brasil y Suecia del 25\% y en México 39\% (Arreguín, et al., 1997). En las ciudades donde es escasa la disponibilidad de agua es imperioso disminuir las cantidades de agua que se pierden por este concepto.

Si bien, en las redes de agua potable no se puede evitar que existan fugas, es necesario llevar a cabo acciones permanentes encaminadas a disminuir el número de fugas y los caudales de las mismas. Una de las complicaciones de la detección de fugas es que la mayoría de éstas no se encuentran visibles, por tanto, para reducirlas es necesario contar con alguna herramienta que permita estimar su localización, así como sus caudales.
Las mediciones de gasto en una red de tuberías de agua potable son complicadas de realizar, mientras que las mediciones de presión son menos difíciles y económicas por lo que el método propuesto se apoya en las mediciones de las cargas de presión en los sitios donde se unen dos o más tuberías (nudos de cruce), además de las características de la red (diámetro, longitud, material, etc.) y los gastos de demanda. La solución óptima del sistema de ecuaciones que se genera al establecer la representación matemática del funcionamiento hidráulico de la red se obtiene mediante la aplicación de un algoritmo genético, los cuales en los últimos años se han utilizado ampliamente en varios campos de la ciencia.

Para estimar la bondad del método propuesto para detectar fugas en redes de tuberías, se realizaron pruebas experimentales en una red de tuberías en el laboratorio de hidráulica del Instituto de Ingeniería de la Universidad Nacional Autónoma de México (UNAM), la cual permite representar algunas condiciones de operación de las redes de agua potable reales. En esta instalación se simula el funcionamiento hidráulico de las tuberías de una manera controlada de presiones y gastos facilitando sus mediciones. Con los datos experimentales se realizó la estimación de fugas, obteniendo buenos resultados.

\section{Planteamiento del problema}

Para simplificar la descripción del método propuesto considérese la red de tuberías cerrada de la figura 1 . Se han numerado los nudos y las tuberías, distinguiendo a estos últimos con números encerrados en un círculo. 
También aparecen con flechas los gastos (q) que egresan (gastos de demanda) o ingresan a la red, por ejemplo, desde algún pozo, los gastos (Q) que fluyen en las tuberías (se han supuesto los sentidos del flujo del gasto) y los caudales de las fugas $\left(Q_{f}\right)$. Se considera que esta red tiene dos fugas, la primera a una distancia $L_{1}$ en el tubo 1 y otra, a la distancia $\mathrm{L}_{2}$ en la tubería 4 . Las dos distancias son desconocidas (pero la longitud de la tubería no) y se encuentran marcadas en la figura. El tramo del nudo 1 al nudo de la fuga 1 , se ha representado como la tubería 1' y el tramo del nudo de la fuga 1 al nudo dos como tubería 1; se hace la misma consideración para la otra fuga. Se conocen las cargas de presión en los nudos del 1 al 5, los diámetros de las tuberías, la longitud de las tuberías, los gastos de demanda en los nudos y el gasto que ingresa a la red $\left(Q_{6}\right)$.

En el sitio donde se juntan varias tuberías se establece que la suma de los gastos que llegan a él es igual a la suma de los gastos que salen del mismo. Así, al aplicar el principio de continuidad en los nudos 1 al 4 y en los nudos de fuga, se establece el sistema de ecuaciones siguiente:

$$
\begin{aligned}
& -Q_{1^{\prime}}-Q_{2}+Q_{6}=q_{1} \\
& Q_{1^{\prime}}-Q_{3}-Q_{4}=-q_{2} \\
& Q_{2^{\prime}}+Q_{3}-Q_{5}=q_{3} \\
& Q_{5}+Q_{4^{\prime}}=q_{4}
\end{aligned}
$$

Al expresar estas ecuaciones en función de la carga de presión, se tiene

$$
\begin{gathered}
-k_{1}\left(h_{1}-h_{f 1}\right)^{1 / 2}-k_{2}\left(h_{1}-h_{3}\right)^{1 / 2}+q_{6}=q_{1} \\
k_{1}\left(h_{f 1}-h_{2}\right)^{1 / 2}-k_{3}\left(h_{2}-h_{3}\right)^{1 / 2}-k_{4}\left(h_{2}-h_{f 2}\right)^{1 / 2}=q_{2} \\
k_{2}\left(h_{1}-h_{3}\right)^{1 / 2}+k_{3}\left(h_{2}-h_{3}\right)^{1 / 2}-k_{5}\left(h_{3}-h_{4}\right)^{1 / 2}=q_{3} \\
k_{5}\left(h_{3}-h_{4}\right)^{1 / 2}+k_{4}\left(h_{f 2}-h_{4}\right)^{1 / 2}=q_{4}
\end{gathered}
$$

donde $k_{i}$ es un coeficiente donde están incluidas la longitud, el diámetro, la aceleración de la gravedad, el factor de fricción, etcétera, de cada tubería. Este sistema

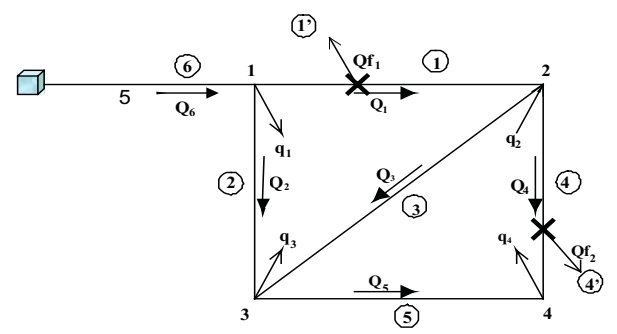

Figura 1. Red de tuberías de ecuaciones tiene como incógnitas a las cargas de presión en los nudos de fuga $h_{f 1}$ y $h_{f 2}$ así como a $k_{1}$ y $k_{2^{\prime}}$.

Se sabe que

$$
k=\left(\frac{1}{C}\right)^{1 / 2} \quad \mathrm{y} \quad C=0.0826 \frac{f L}{d^{5}}
$$

por lo que el sistema de ecuaciones [2] se puede escribir así:

$$
\begin{gathered}
\left(\frac{\beta_{1}}{L_{1^{\prime}}}\right)^{1 / 2}\left(h_{1}-h_{f 1}\right)^{1 / 2}-\left(\begin{array}{c}
\beta_{2} \\
L_{2}
\end{array}\right)^{1 / 2}\left(h_{1}-h_{3}\right)^{1 / 2}=q_{1}-q_{6} \\
\left(\begin{array}{c}
\beta_{1} \\
L_{1}-L_{1^{\prime}}
\end{array}\right)^{1 / 2}\left(h_{f 1}-h_{2}\right)^{1 / 2}-\left(\begin{array}{c}
\beta_{3} \\
L_{3}
\end{array}\right)^{1 / 2}\left(h_{2}-h_{3}\right)^{1 / 2}-\left(\begin{array}{c}
\beta_{4} \\
L_{4^{\prime}}
\end{array}\right)^{1 / 2}\left(h_{2}-h_{f 2}\right)^{1 / 2}=q_{2} \\
\left(\frac{\beta_{5}}{L_{5}}\right)^{1 / 2}\left(h_{3}-h_{4}\right)^{1 / 2}+\left(\frac{\beta_{4}}{L_{4}-L_{4^{\prime}}}\right)\left(h_{f 2}-h_{4}\right)^{1 / 2}=q_{4}
\end{gathered}
$$

donde

$$
\beta_{i}=\frac{d^{5}}{0.0826 f_{i}}
$$

Finalmente, para el ejemplo mostrado en la figura 1, se establece un sistema de tres ecuaciones no lineales con cuatro incógnitas. Las incógnitas serían por tanto, las longitudes a partir del primer nudo en sentido del flujo, donde se encuentra la fuga y las cargas en estos puntos, es decir, la carga de presión en donde se localiza la fuga, la cual permite obtener el caudal. Para resolver este sistema de ecuaciones de manera que las cargas de presión sean parecidas a las medidas se propone en este trabajo emplear un algoritmo genético, ya que es un método robusto y eficiente para esta clase de problemas matemáticos.

La solución propuesta con este algoritmo se evalúa verificando el funcionamiento hidráulico de la red para comprobar que la solución propuesta sea el óptimo global, o bien, un valor muy cercano a él. Esto se realiza con el cálculo de las cargas en cada nudo, a partir de las posiciones y caudales de fuga propuestos por el algoritmo genético.

Los estudios de las redes de tuberías funcionando a presión se suelen realizar considerando flujo permanente; es decir, sin cambios a lo largo del tiempo.

\section{Algoritmos genéticos}

El objetivo de las técnicas de optimación es encontrar el óptimo global (o los óptimos globales) de una función. Desgraciadamente, sólo en algunos casos limitados, puede garantizarse convergencia hacia el óptimo glo- 
bal. De hecho, la mayoría de las técnicas usadas para optimación localizan óptimos locales, pero no puede garantizarse convergencia al óptimo global a menos que se usen técnicas exhaustivas.

El gran campo de aplicación de los AG's se relaciona con aquellos problemas para los cuales no existen técnicas especializadas (Rodríguez et al., 2004).

Los algoritmos genéticos, inspirados en la selección natural y la genética, son técnicas de optimación que tienen la ventaja de realizar la búsqueda sobre un conjunto de posibles alternativas simultáneamente (sobre una población), y no a partir de un solo punto, lo cual reduce la posibilidad de converger a un óptimo local. Estos algoritmos parten de una población de soluciones generadas, inicialmente, en forma aleatoria, las cuales se van adaptando al problema (mejorando) a través de las generaciones mediante la selección de las más aptas y la aplicación de operadores genéticos como son cruza y mutación sobre estas soluciones. De esta manera se crea una nueva generación con tendencias a moverse hacia el óptimo al paso del tiempo (generaciones).

Los algoritmos genéticos son procedimientos adaptativos (basados en sistemas de analogías con el funcionamiento de la naturaleza y la evolución de la misma) que pueden usarse para resolver problema de optimación, búsqueda y clasificación, dentro de los cuales tenemos sistemas de ecuaciones no lineales. Es una técnica robusta basada en el proceso genético de los organismos vivos.

En los AG's se necesita una codificación de cada una de las posibles soluciones (individuos). Además, se requiere de una función de evaluación de aptitud, que asigna un valor de calidad a cada solución. Durante la ejecución del algoritmo, los padres deben ser seleccionados para la reproducción; luego, dichos padres se cruzarán para generar hijos, sobre cada uno de los cuales actuará el operador de mutación. Como resultado, los hijos forman un conjunto de individuos más apto (valores más cercanos a la solución del problema), que constituyen la población de la siguiente generación.

Los principios básicos de los AG's fueron establecidos por Holland en 1975. Si bien, es difícil garantizar que con este algoritmo se encuentre la solución del problema, existe evidencia empírica de que se obtienen soluciones aceptables en un tiempo de cómputo relativamente corto.

Para resolver el sistema no lineal (ecuaciones 3), se plantea un algoritmo general simple, de la siguiente manera:

- Población de soluciones. El algoritmo genera de forma aleatoria un conjunto de soluciones del problema que serán evaluadas. Cada una de las soluciones (individuo) depende del número de tuberías con fuga de la red y la longitud del cromosoma del individuo está en función del número de variables y del valor de la precisión para cada una. Para el ejemplo que se muestra en este trabajo se tienen 18 tuberías con fuga (figura 2) y como variables se establecieron las cargas de presión y la longitud donde se presenta la fuga a partir del nudo de cruce.

- Asignación de una calificación a cada individuo. Se establece una función objetivo con dos penalizaciones que se denomina de aptitud, para asignar a cada una de las soluciones una calificación. En este caso se empleó la siguiente:

$$
F_{a}=\Sigma\left|q_{\text {medido }}-q_{\text {estimado }}\right|+p_{1} k n+p_{2} n
$$

donde $p_{1} \mathrm{y} p_{2}$ son valores de penalización asociados a las soluciones encontradas por el algoritmo. $p_{1}$ se establece en los casos donde esta solución da como resultado una fuga negativa en alguna tubería de la red; $p_{2}$ en el caso de tener un gasto de demanda negativo. $k$ es una constante con valor de 100 para darle mayor peso a las soluciones donde se presenten $n$ fugas negativas en la red. Este término $n$ se introduce en el segundo y tercer término de la función objetivo para considerar el caso cuando la mejor solución no presenta fugas negativas, pero sí considere aquellos casos donde los gastos de demanda sean negativos, lo cual puede suceder, es decir, se presenta un ingreso a la red.

- Mezclado de las mejores soluciones para generar otras que en promedio sean superiores. Una vez que se estimó la aptitud de cada individuo de la población, se seleccionan parejas para llevar a cabo la cruza y generar otra población (generación), la cual se evaluará nuevamente. El método de selección para la cruza empleado fue el de ruleta (Goldberg, 1989), con un valor de 0.6 de cruza y de 0.01 de mutación.

El método es general y capaz de ser aplicado a distintos tipos de problemas de optimación, con la ventaja de que son fáciles de plantear y sencillos de codificar en la computadora.

Se utilizó el algoritmo genético simple implantado en Matlab (MathWorks,1992) con algunas modificaciones en los archivos de ejecución. En la aplicación del algoritmo genético simple se utilizaron en cada corrida 
150 generaciones (iteraciones) y el número de individuos elegido fue de 2000, ya que para estos valores los resultados obtenidos se aproximaban a la solución buscada.

Una vez obtenida la solución óptima, estableciendo que en la red cada tubería puede tener sólo una fuga, con los datos respecto a posición de fuga y carga de presión en el nudo de fuga o caudal de la misma, se realiza el cálculo hidráulico de la red para comparar la diferencia entre los datos encontrados con el algoritmo genético y la modelación numérica de la red. Este proceso se realizó 8 veces, llegando a una solución con poco error en las cargas de presión en los nudos de fuga calculados y los estimados con el algoritmo.

\section{Aspectos adicionales}

En el método se establecieron las siguientes hipótesis:

a) El número de fugas por tubo es uno (si se tiene la sospecha de la existencia de más de una fuga en la tubería, convendría dividir las tuberías, sobre todo las más largas en varias tuberías de longitud menor y conectadas en serie, siendo aplicable el método propuesto sin ninguna restricción adicional).

b) Se cuenta con mediciones de la carga de presión en varios nudos de la red de tuberías (nudos de cruce).

c) Se conocen los gastos de demanda y el gasto total que se ingresa a la red de tuberías.

d) Se conoce el factor de fricción de cada tubo de red.

f) El flujo en la red es permanente (debido a la variación de la demanda de los usuarios de la red de distribución de agua potable el flujo de agua dentro de las tuberías es no permanente. Para detectar por lo menos una fuga de agua en las tuberías o en las conexiones, es recomendable considerar como condición de funcionamiento, con flujo permanente, el momento, casi siempre en la noche, cuando es más baja la demanda, para que se tengan cargas de presión más grandes, y por ende, mayores gastos de fuga.

\section{Ejemplo de aplicación}

Para verificar la robustez de este método se realizaron pruebas en la red de distribución del Laboratorio de Hidráulica del Instituto de Ingeniería, UNAM; ésta tiene las características mostradas en la figura 2, en ella se han incluido los valores de los gastos de demanda y de fuga, además de las cargas de presiones. Para aplicar el método expuesto en este trabajo se consideró que las fugas se presentan en los nudos 2, 4, 6, 8, 10, 12, 14, 16,

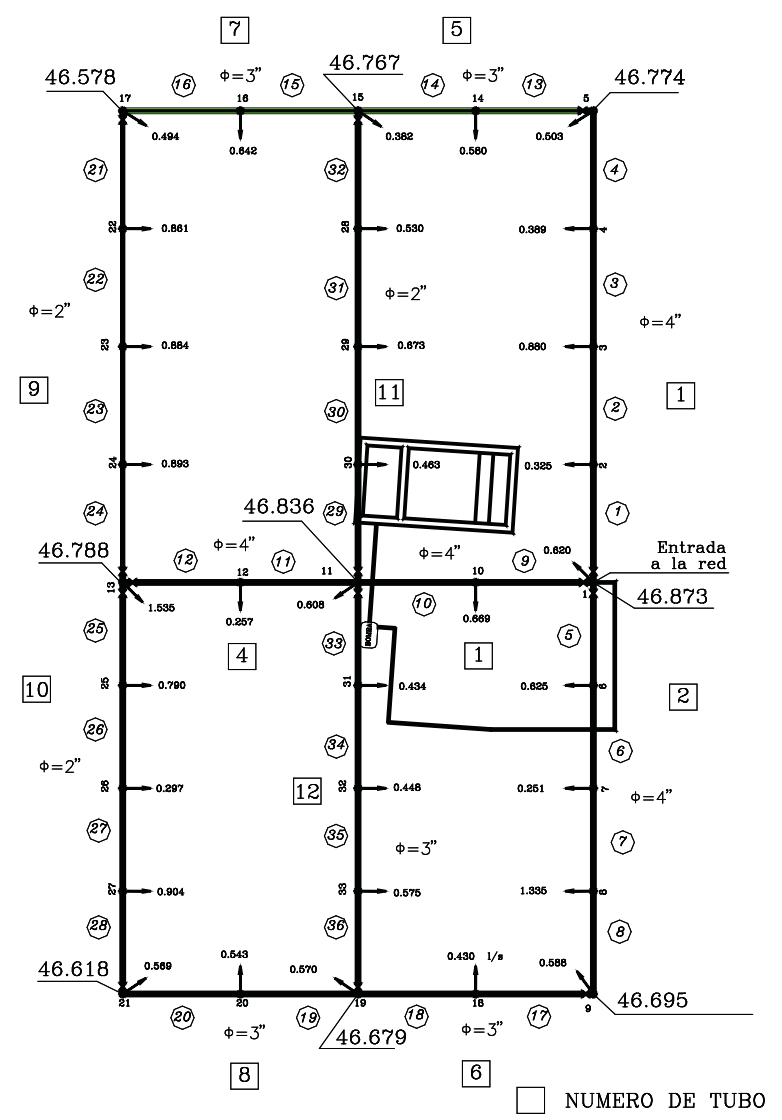

Figura 2. Red de tuberías del Laboratorio de Hidráulica del Instituto de Ingeniería, UNAM (sin fugas)

$18,20,22,24,25,27,28,3031,33$. Por lo tanto, el problema es analizar una red de 18 tuberías y 15 nudos, con las características mostradas en la tabla 1 y determinar, dadas las cargas de presión medidas en los nudos de cruce, las tuberías en las cuales se presenta una fuga, la posición de ésta y el caudal de fuga. La red de distribución del Laboratorio de Hidráulica del Instituto de Ingeniería de la UNAM tiene las características mostradas en la figura 2, en ella se han incluido los valores de gastos y cargas de presiones cuando no se presentan ningún gasto de fuga.

a) Medición de los gastos de salida de la red. Se midieron los gastos de salida en los nudos de la red que corresponden a los gastos de demanda teórica.

b) Mediciones de presión sin fugas. En el dispositivo físico se obtuvieron las mediciones de presión mostradas en la figura 2. En ella también se anotaron los gastos de demanda teórica

c) Calibración de la red de tuberías experimental. Con las mediciones de la carga de presión y de los gastos 
se calibró la red de tuberías. Ello permitió estimar los factores de fricción de Darcy que están consignados en la tabla 1 junto con algunas características de la red.

Los factores de fricción incluyen una cantidad adicional del orden del valor del factor de fricción equivalente al coeficiente de pérdida local originada por codos, válvulas, placas de orificio empleadas para medir gastos a lo largo de las tuberías y otros accesorios existentes en la red experimental por lo que aparecen valores mayores a los que se obtienen con sólo fricción

d) Estimación de demandas cuando existen fugas. Se obtuvieron las demandas para el momento en que se realizan las mediciones de presión para detectar fugas.

Tabla 1. Características de la red experimental

\begin{tabular}{|c|c|c|c|c|c|}
\hline Tubería & $\begin{array}{c}\text { De } \\
\text { nudo }\end{array}$ & $\begin{array}{c}\text { A } \\
\text { nudo }\end{array}$ & $\begin{array}{l}\text { Longitud } \\
(\mathrm{m})\end{array}$ & $\begin{array}{c}\text { D } \\
(\mathrm{plg})\end{array}$ & $\mathrm{f}$ \\
\hline 1 & 1 & 2 & 2.75 & 4 & 0.0744 \\
\hline 2 & 2 & 3 & 2.75 & 4 & 0.0583 \\
\hline 3 & 2 & 3 & 2.75 & 4 & 0.0582 \\
\hline 4 & 4 & 5 & 2.85 & 4 & 0.0616 \\
\hline 5 & 1 & 6 & 2.4 & 4 & 0.1443 \\
\hline 6 & 6 & 7 & 2.4 & 4 & 0.1126 \\
\hline 7 & 7 & 8 & 2.4 & 4 & 0.1123 \\
\hline 8 & 8 & 9 & 2.4 & 4 & 0.1190 \\
\hline 9 & 1 & 10 & 2.75 & 4 & 0.0104 \\
\hline 10 & 10 & 11 & 2.75 & 4 & 0.0100 \\
\hline 11 & 11 & 12 & 2.75 & 4 & 0.0425 \\
\hline 12 & 12 & 13 & 2.75 & 4 & 0.0515 \\
\hline 13 & 5 & 14 & 2.75 & 3 & 0.0052 \\
\hline 14 & 14 & 15 & 2.75 & 3 & 0.0058 \\
\hline 15 & 15 & 16 & 2.75 & 3 & 0.3486 \\
\hline 16 & 16 & 17 & 2.75 & 3 & 0.6908 \\
\hline 17 & 9 & 18 & 2.75 & 3 & 0.0180 \\
\hline 18 & 18 & 19 & 2.75 & 3 & 0.0165 \\
\hline 19 & 19 & 20 & 2.75 & 3 & 0.1150 \\
\hline 20 & 20 & 21 & 2.75 & 3 & 0.2322 \\
\hline 21 & 17 & 22 & 2.75 & 2 & 0.1111 \\
\hline 22 & 22 & 23 & 2.75 & 2 & 1.9669 \\
\hline 23 & 23 & 24 & 2.75 & 2 & 0.0586 \\
\hline 24 & 24 & 13 & 2.75 & 2 & 0.0647 \\
\hline 25 & 13 & 25 & 2.4 & 2 & 0.0323 \\
\hline 26 & 25 & 26 & 2.4 & 2 & 0.0396 \\
\hline 27 & 26 & 27 & 2.4 & 2 & 3.9574 \\
\hline 28 & 27 & 21 & 2.4 & 2 & 0.0567 \\
\hline 29 & 11 & 30 & 2.75 & 2 & 0.0140 \\
\hline 30 & 30 & 29 & 2.75 & 2 & 0.0105 \\
\hline 31 & 29 & 28 & 2.75 & 2 & 0.3564 \\
\hline 32 & 28 & 15 & 2.75 & 2 & 0.0225 \\
\hline 33 & 11 & 31 & 2.4 & 3 & 0.1062 \\
\hline 34 & 31 & 32 & 2.4 & 3 & 0.0976 \\
\hline 35 & 32 & 33 & 2.4 & 3 & 0.3004 \\
\hline 36 & 33 & 19 & 2.4 & 3 & 14.9882 \\
\hline
\end{tabular}

Con las cargas de presión se formó un sistema de 15 ecuaciones, donde las incógnitas son la distancia donde se localiza la fuga para cada tubería (si existe), la carga de presión de este punto y por tanto, el caudal de fuga.

Con el algoritmo genético se procedió a buscar la solución, empleando 2000 individuos, 150 generaciones y una probabilidad de cruza de 0.6 (probabilidad de aplicar el operador de cruza; de lo contrario, los individuos pasan sin modificación a la siguiente generación). Además se considera la mutación con una probabilidad de 0.01, probabilidad baja haciendo una analogía con la probabilidad de mutación en sistemas naturales. La mutación tiene la finalidad de inyectarle diversidad a la población y con esto reducir la posibilidad de una convergencia prematura (óptimo local).

Una vez establecida la solución mejor que determinaba el algoritmo, ésta se evaluaba hidráulicamente, de forma que con las distancias y caudales de fuga, se determinan las cargas de presión en todos los nudos (inclusive los de fuga), para así evaluar las diferencias entre los valores medidos y los calculados.

Al verificar la diferencia entre los valores de cargas de presiones, para cada solución (para cada tubo) se determinó un factor de error en función de la diferencia de las mismas, afectadas por un peso establecido en función de diferencias de gastos por unidad de diferencia de carga de presión medida entre cada nudo en donde se busca una fuga. Este factor se establece para diferenciar entre las soluciones la relevancia que tiene entre una y otra la diferencia de una unidad en carga de presión, por ejemplo, para el espacio de búsqueda de la fuga entre el nudo 1 y 3, éste se encuentra entre 46.854 y $46.788 \mathrm{~m}$, a diferencia, por ejemplo, para la que se presenta entre el nudo 3 y el 5, donde la búsqueda sería entre 46.788 y $46.755 \mathrm{~m}$.

\section{Resultados}

De acuerdo con los resultados obtenidos se verifica que el algoritmo genético utilizado es robusto y permite ubicar las fugas y determinar sus gastos, entendiendo que se trata de un procedimiento aleatorio, con lo que se puede obtener respuestas distintas y no se garantiza que se haya llegado al óptimo global en una corrida, de ahí la ventaja de proponer e implementar la verificación del comportamiento hidráulico con la solución propuesta.

Después de evaluar 8 corridas, conjuntamente el algoritmo genético y el programa del método del instituto, la aptitud de la función objetivo fue menor a $1 \times 10^{-4}$, que aunque parezca bajo, depende del problema que se esté abordando y sobre todo, de las variables considera- 
das y la función matemática a optimar. El valor de los caudales de fuga que se manejaron en la red del laboratorio son muy pequeños y la diferencia de cargas de presión entre los nudos es además del orden de centímetro o milímetro. Por tal razón, no existe diferencia significativa entre las posiciones establecidas y por tanto, los caudales registrados.

Pese a esta situación los resultados presentados en la tabla 2 son robustos, ya que de las 18 fugas solamente una no la sitúa en el lugar correcto (fuga 8), su posición la marca a una distancia de $1.75 \mathrm{~m}$, a diferencia del resto que las sitúa correctamente; y por tanto, al analizar su funcionamiento hidráulico, las cargas de presión calculadas, difieren de las medidas, pero este error es pequeño, ya que se puede apreciar en esta tabla, que el

Tabla 2. Resultados obtenidos

\begin{tabular}{|c|c|c|c|c|c|c|c|c|c|}
\hline Nudo & Med. & AGR1 & AGR2 & AGR3 & AGR6 & AGR7 & AGR8 & C. R. R. & \%error \\
\hline H1 & 46.854 & & & & & & & 46.854 & 0.0000 \\
\hline F1 & & 46.815 & 46.815 & 46.815 & 46.815 & 46.815 & 46.815 & 46.815 & 0.0000 \\
\hline $\mathrm{H} 2$ & 46.788 & & & & & & & 46.788 & 0.0000 \\
\hline F2 & & 46.770 & 46.770 & 46.770 & 46.770 & 46.770 & 46.770 & 46.770 & 0.0000 \\
\hline $\mathrm{H} 3$ & 46.752 & & & & & & & 46.755 & 6.4164 \\
\hline F3 & & 46.778 & 46.779 & 46.778 & 46.779 & 46.779 & 46.779 & 46.781 & 0.0043 \\
\hline $\mathrm{H} 4$ & 46.736 & & & & & & & 46.736 & 0.0000 \\
\hline F4 & & 46.699 & 46.697 & 46.699 & 46.697 & 46.697 & 46.697 & 46.696 & 2.1415 \\
\hline $\mathrm{H} 5$ & 46.679 & & & & & & & 46.676 & 6.4273 \\
\hline F5 & & 46.834 & 46.834 & 46.834 & 46.834 & 46.834 & 46.834 & 46.834 & 0.0000 \\
\hline H6 & 46.810 & & & & & & & 46.817 & 14.9518 \\
\hline F6 & & 46.794 & 46.794 & 46.794 & 46.794 & 46.794 & 46.794 & 46.794 & 0.0000 \\
\hline $\mathrm{H} 7$ & 46.754 & & & & & & & 46.769 & 32.0725 \\
\hline F7 & & 46.751 & 46.751 & 46.751 & 46.751 & 46.751 & 46.751 & 46.751 & 0.0000 \\
\hline H8 & 46.745 & & & & & & & 46.748 & 6.4174 \\
\hline F8 & & 46.629 & 46.629 & 46.660 & 46.660 & 46.660 & 46.660 & 46.645 & 32.1578 \\
\hline H9 & 46.555 & & & & & & & 46.559 & 8.5912 \\
\hline F9 & & 46.669 & 46.666 & 46.669 & 46.666 & 46.666 & 46.666 & 46.666 & 0.0000 \\
\hline $\mathrm{H} 10$ & 46.663 & & & & & & & 46.660 & 6.4295 \\
\hline F10 & & 46.644 & 46.637 & 46.643 & 46.637 & 46.637 & 46.630 & 46.628 & 4.2893 \\
\hline H11 & 46.597 & & & & & & & 46.598 & 2.1460 \\
\hline F11 & & 46.535 & 46.534 & 46.532 & 46.532 & 46.532 & 46.532 & 46.523 & 19.3453 \\
\hline $\mathrm{H} 12$ & 46.561 & & & & & & & 46.561 & 0.0000 \\
\hline F12 & & 46.602 & 46.603 & 46.603 & 46.603 & 46.603 & 46.603 & 46.604 & 2.1457 \\
\hline F13 & & 46.737 & 46.735 & 46.737 & 46.735 & 46.735 & 46.735 & 46.735 & 0.0000 \\
\hline H13 & 46.728 & & & & & & & 46.728 & 0.0000 \\
\hline F14 & & 46.574 & 46.576 & 46.575 & 46.576 & 46.576 & 46.576 & 46.583 & 15.0269 \\
\hline F16 & & 46.748 & 46.748 & 46.749 & 46.749 & 46.749 & 46.749 & 46.748 & 2.1391 \\
\hline H14 & 46.786 & & & & & & & 46.786 & 0.0000 \\
\hline F15 & & 46.796 & 46.796 & 46.794 & 46.794 & 46.794 & 46.794 & 46.795 & 2.1370 \\
\hline F17 & & 46.790 & 46.790 & 46.792 & 46.792 & 46.792 & 46.792 & 46.792 & 0.0000 \\
\hline H15 & 46.779 & & & & & & & 46.779 & 0.0000 \\
\hline F18 & & 46.770 & 46.766 & 46.762 & 46.762 & 46.762 & 46.762 & 46.761 & 2.1385 \\
\hline
\end{tabular}

error máximo que se presenta en esta fuga es de 32\%. Además, se verificó la suma de los gastos (suministrados y de fuga), para verificar el error en ellos, dando una diferencia menor a un centilitro.

De los resultados del ejemplo de aplicación, se nota que cuando la magnitud de las fugas no son grandes en comparación con las demandas, los resultados del método dan cierta aproximación a las fugas. No obstante, con sus posibles limitaciones, el método descrito es perfectible y puede ser empleado junto con los instrumentos de detección de fugas (geófonos, varillas de sondeo, equipo electrónico) para que sea más eficaz su ubicación y proceder a realizar las reparaciones con una mayor garantía de éxito.

\section{Comentarios y conclusiones}

Con base en los resultados obtenidos en este trabajo y en otros similares, se confirma que los algoritmos genéticos son herramientas robustas para esta clase de problemas de optimación, y que los valores obtenidos tienen una adecuada precisión, ya que el porcentaje de error fue bajo.

Por otro lado, aún cuando estos resultados son alentadores, será necesario aplicar el procedimiento propuesto en redes de mayor tamaño para comprobar su eficacia, y determinar pesos o factores que permitan elegir y acotar los espacios de solución, implementando el funcionamiento hidráulico de la red, consideración muy importante para el caso de redes grandes y complicadas. 


\section{Referencias}

Arreguín F., Ochoa L. y Fernández A. Evaluación de pérdidas en redes de distribución de agua. TLALOC-AMH, Órgano informativo de la Asociación Mexicana de Hidráulica (AMH), No.10. 1997.

Goldberg D.E. Genetic Algorithms in Search, Optimization and Machina Learning, Addison-Wesley. 1989.

Holland J.H. Adaptation in Natural and Artificial Systems, University of Michigan Press. 1975.

Rodríguez V.K., Jiménez M.M. R. y Fuentes M.O.A. Los algoritmos genéticos en la Ingeniería de los sistemas de abastecimiento ¿la modelación del futuro de las redes de distribución? XVIII Congreso Nacional de Hidráulica, San Luis Potosí, S. L. P. , México, 2004. Tema 11: Agua potable y alcantarillado, P. 1533.

\section{Bibliografía sugerida}

Fuentes M.O.A., Palma N.A., Rivera T.F.G. y Rodríguez V.K. Localización de fugas y determinación de sus gastos en una red de tuberías de agua potable usando un algoritmo genético. XVIII Congreso Nacional de Hidráulica, San Luis Potosí, S. L. P., México, 2004. Tema 11: Agua potable y alcantarillado. P. 1557.

Fuentes M.O.A., Rodríguez V.K., Jímenez M.M.R.,De Luna C.F. y Vega S.B.E. Método para la detección de fugas en redes de distribución de agua potable. Memorias del $3^{\text {er }}$ Seminario Hispano-Brasileño sobre Planificación, Proyecto y Operación de redes de abastecimiento de agua, Universidad Politécnica de Valencia, España. 2004.

López A., Vela F., Iglesias P. Análisis de seguridad en abastecimientos y su aplicación a la detección de fugas. Ingeniería del agua, 2(3). 1995.

Sánchez J. y Fuentes O. Método para detectar fugas mayores en una red de agua potable. Ingeniería del agua, 3(1). 1996.

Luvizotto E. y Ocampos A. Compando los métodos de Levembenvereg e Nelder-Mead em modelos de deteccao de fuga. Seminario Hispano-Brasileiro sobre planificación, proyecto $\mathrm{y}$ operación de redes de abastecimiento de agua. Valencia, España. 2002. Pp. 451-463.

Pudar S., Ligget A. Leaks in Pipe Networks. Journal of Hydraulics Engineering, ASCE, 118(7). 1992.

Sánchez J. y Fuentes O. Actualización de un método para calcular redes de tuberías funcionando a presión en régimen permanente. Revista de Ingeniería, LXI, México DF. 1991.

Sánchez B. y Fuentes M. Método para detectar fugas mayores en una red de agua potable. Revista Ingeniería del Agua, Universidad Politécnica de Valencia, España, 3(1), marzo 1996, ISNN 1134-2196.

Vela A., Martínez F., García-Serra J. y Pérez R. Estrategias óptimas para la reducción de pérdidas de agua en sistemas de abastecimiento. Ingeniería del Agua, 1(1), Valencia, España. 1994.

Vítovský J., Simpson A. Leak Detection and Calibration Using Transients and Genetic Algorithms. Journal of Water Resources Planning and Management, 126(4):262-265. 2000

\section{Semblanza de los autores}

Óscar A. Fuentes-Mariles. Es ingeniero civil egresado de la Facultad de Ingeniería, UNAM, con maestría y doctorado en hidráulica por la misma institución. Actualmente es investigador de la Coordinación de Hidráulica del Instituto de Ingeniería donde ha colaborado desde 1973, desde ese año, ha sido profesor de asignatura en la Facultad y Posgrado de Ingeniería de la UNAM. Se destaca por sus aportaciones a los métodos numéricos aplicados a la hidráulica, hidráulica marítima, hidráulica fluvial, además de su participación en el Centro Nacional de Prevención de Desastres.

Adriana Palma-Nava. Obtuvo el grado de ingeniería civil en la Facultad de Ingeniería, UNAM, con maestría en hidráulica y candidata a doctorado por la misma institución. Actualmente colabora en proyectos de investigación patrocinados en la Coordinación de Hidráulica del Instituto de Ingeniería. Desde 1996, se ha desempeñado como profesora de asignatura en la Facultad de Ingeniería y en la Facultad de Contaduría y Administración de la
UNAM. Se destaca por sus aportaciones al desarrollo de modelos numéricos de acuíferos, en particular el del Valle de México, participando en el desarrollo del mismo desde el año 2000.

Katya Rodríguez-Vázquez. Obtuvo el grado de ingeniería en computación en 1994 por la Facultad de Ingeniería, UNAM y el doctorado por la Universidad de Sheffield, Reino Unido en 1999. Desde abril de 1999, está adscrita al Instituto de Investigaciones en Matemáticas Aplicada y en Sistemas (IIMAS), UNAM, siendo investigadora Titular "A". Sus líneas de interés se enfocan al cómputo evolutivo y bioinspirado, así como en los algoritmos evolutivos multicriterio (optimización multiobjetivo). Las aplicaciones han sido diversas desde la ingeniería civil hasta la biología y economía. Ha publicado artículos en revistas y conferencias tanto nacionales como internacionales y ha formado parte de comités técnicos de congresos de áreas afines a sus líneas de investigación, así como revisora en revistas arbitradas internacionales. Participa activamente en el programa de posgrado en ciencia e ingeniería de la computación, UNAM. 